\title{
NKT cells in mucosal immunity
}

\author{
S Middendorp ${ }^{1}$ and EES Nieuwenhuis ${ }^{1}$
}

The gastrointestinal tract allows the residence of an almost enumerable number of bacteria. To maintain homeostasis, the mucosal immune system must remain tolerant to the commensal microbiota and eradicate pathogenic bacteria. Aberrant interactions between the mucosal immune cells and the microbiota have been implicated in the pathogenesis of inflammatory disorders, such as inflammatory bowel disease (IBD). In this review, we discuss the role of natural killer T cells (NKT cells) in intestinal immunology. NKT cells are a subset of non-conventional T cells recognizing endogenous and/or exogenous glycolipid antigens when presented by the major histocompatibility complex (MHC) class I-like antigen-presenting molecules CD1d and MR1. Upon T-cell receptor (TCR) engagement, NKT cells can rapidly produce various cytokines that have important roles in mucosal immunity. Our understanding of NKT-cell-mediated pathways including the identification of specific antigens is expanding. This knowledge will facilitate the development of NKT cellbased interventions and immune therapies for human intestinal diseases.

\section{INTRODUCTION}

The main function of the intestine is digestion and absorption of nutrients from food and recovery of water and electrolytes. In addition, the intestine houses a large immune compartment, as it is responsible for prevention and control of mucosal infections, regulation of microbial colonization, and induction of oral tolerance. Impaired or uncontrolled mucosal immune responses toward commensal or pathogenic bacteria may result in infections and are implicated in the pathogenesis of autoimmune diseases, such as inflammatory bowel disease (IBD).

Natural killer T cells (NKT cells) represent a minor subset of T lymphocytes that share cell-surface proteins with conventional T cells and NK cells. ${ }^{1}$ These cells are located mainly in the liver, spleen, and bone marrow and depend on the thymus for their development, similar to conventional T cells. Many NKT cells express a T-cell receptor (TCR) that recognizes lipid antigens presented by the non-classical major histocompatibility complex (MHC) class I molecule CD1d. In the intestine, variant and invariant NKT (iNKT) cells are found among intraepithelial lymphocytes (IELs) and within the lamina propria (LP).

The activation of intestinal NKT cells by CD1d-expressing antigen-presenting cells (APCs) may contribute to mucosal immunity directed against pathogenic or commensal bacteria. On the other hand, uncontrolled or insufficient activation of NKT cells may have an important role in the pathogenesis of IBD.
With reference to recent literature on the role of iNKT cells in mucosal immunology, ${ }^{2-4}$ this review will mainly focus on the role of other intestinal NKT cells (e.g., mucosal (m) NKT cells and $\gamma \delta \mathrm{NKT}$ cells) in mucosal immunity and the interaction of NKT cells with other immune cells such as dendritic cells (DCs) and B lymphocytes.

\section{NKT cell nomenclature}

NKT cells were originally defined by the co-expression of a conventional $\alpha \beta$ TCR and NK receptors such as NK1.1 in certain mouse strains and CD161 in humans. Similar to NK cells, these cells contain perforin and granzymes, enabling them to take part in innate immune responses. ${ }^{1,5,6}$

Unlike conventional $\mathrm{T}$ cells that recognize peptides bound to MHC class I or II molecules, NKT cells recognize glycolipid structures that are presented by CD1d. Most NKT cells express an invariant TCR, as it consists of invariant $\alpha$-chains combined with a restricted subset of $\beta$-chains. Ligation of the NKT-cellspecific TCR leads to rapid secretion of cytokines, including interferon- $\gamma($ IFN- $\gamma)$, interleukin-4 (IL-4), and various chemokines. ${ }^{1}$ Expanding knowledge on the properties of NKT cells gave rise to a new classification according to the (in)-variance of the involved TCR $\alpha$-chain, the selecting antigen-presenting molecule, and the expression of various cell surface molecules. ${ }^{3}$

There are so-called canonical or type I NKT cells, including both iNKT and mNKT (or mucosal-associated invariant

\footnotetext{
${ }^{1}$ Department of Pediatric Gastroenterology and Laboratory of Pediatrics, Erasmus MC Sophia Children's Hospital, University Medical Center, Rotterdam,

The Netherlands. Correspondence: EES Nieuwenhuis (e.nieuwenhuis@erasmusmc.nl) and S Middendorp (s.middendorp@erasmusmc.nl) 
Table 1 Mouse models for studying iNKT cells and their related antigen-presenting molecules

\begin{tabular}{|c|c|c|c|}
\hline & Details & Phenotype & Reference \\
\hline \multicolumn{4}{|l|}{ Transgenic } \\
\hline Va14-Ja18 & $\begin{array}{l}\text { V } \alpha \text {-J } \alpha \text { for } \\
\text { mouse iNKT } \\
\text { TCR }\end{array}$ & iNKT-Tg & Wakao et al. ${ }^{140}$ \\
\hline Va14-Ja281 & $\begin{array}{l}\vee \alpha-J \alpha \text { for } \\
\text { mouse iNKT } \\
\text { TCR }\end{array}$ & iNKT-Tg & $\begin{array}{l}\text { Taniguchi } \\
\text { et al. }{ }^{141}\end{array}$ \\
\hline$V \alpha 24-J a Q-m C \alpha$ & $\begin{array}{l}\text { V } \alpha \text {-J } \alpha \text { for } \\
\text { human iNKT } \\
\text { TCR }\end{array}$ & iNKT-Tg & $\begin{array}{l}\text { Capone } \\
\text { et al. }{ }^{142}\end{array}$ \\
\hline$V \alpha 14 / V \beta 8$ & $\begin{array}{l}V \alpha \text { and } V \beta \text { for } \\
\text { mouse iNKT } \\
\text { TCR }\end{array}$ & iNKT-Tg & Kawano et al. ${ }^{51}$ \\
\hline Va19-Ja33 & $\begin{array}{l}\vee \alpha-J \alpha \text { for } \\
\text { mouse mNKT } \\
\text { TCR }\end{array}$ & mNKT-Tg & $\begin{array}{l}\text { Kawachi } \\
\text { et al. }\end{array}$ \\
\hline \multicolumn{4}{|l|}{ Knockout } \\
\hline$J \alpha 281$ & $\begin{array}{l}\text { Ja chain for } \\
\text { mouse iNKT } \\
\text { TCR }\end{array}$ & No iNKT & Cui et al. ${ }^{143}$ \\
\hline CD1d & $\begin{array}{l}\text { Ag-presenting } \\
\text { molecule for } \\
\text { iNKT cells }\end{array}$ & No iNKT & $\begin{array}{l}\text { Chen } \\
\text { et al. }{ }^{144} \text { and } \\
\text { Smiley et al. } .^{145}\end{array}$ \\
\hline MR1 & $\begin{array}{l}\text { Ag-presenting } \\
\text { molecule for } \\
\text { mNKT cells }\end{array}$ & No mNKT & Treiner et al. ${ }^{9}$ \\
\hline
\end{tabular}

iNKT, invariant natural killer cells; mNKT, mucosal natural killer cells; TCR, T-cell receptor.

T cells (MAIT cells)) cells, which express TCRs with invariant $\mathrm{V} \alpha$ chains. ${ }^{3}$ The TCR of the iNKT cell population in mice contains the Val4 gene segment, whereas its human counterpart uses Va24. Type I iNKT cells can be identified by using $\alpha$-galactosylceramide ( $\alpha$ GalCer)/CD1d tetramers. ${ }^{7,8}$ The mNKT population uses the $\mathrm{V} \alpha 7.2$ gene segment in mice and $\mathrm{V} \alpha 19$ in humans. ${ }^{9}$ Several mouse models have been developed for studying NKT cells and related antigen-presenting molecules (Table 1). The generation of knockout mice and transgenic mice with overexpression of invariant TCRs by iNKT or mNKT cells has greatly facilitated the functional analyses of these cells.

Another CD1d-restricted NKT cell population, known as variant or type II NKT cells, has a variant TCR rearrangement and has been identified in both mice and humans. ${ }^{10,11}$ A major type II NKT subset recognizes the self-glycolipid 3 -sulfated galactosylceramide, known as sulfatide, and can be identified by sulfatide/CD1d tetramers. ${ }^{11}$ Although type II NKT cells have not been studied as extensively as type I NKT cells, these cells have been implicated in a number of autoimmune diseases. Interestingly, high IL-13 secretion by type II NKT cells has been suggested in the pathogenesis of ulcerative colitis. ${ }^{12}$ Recently, it was found that sulfatide-reactive type II NKT cells are able to regulate type I NKT cells by modulating DC function. ${ }^{13,14}$
A small group of T cells co-expressing NK markers are the so-called $\gamma \delta \mathrm{NKT}$ cells, which express a $\gamma \delta \mathrm{TCR}$ instead of an aßTCR. ${ }^{15}$

\section{NKT cell differentiation}

Similar to conventional T cells, iNKT cells develop in the thymus and branch off at the double-positive (DP) stage. In the thymus, DP cells develop into $\mathrm{CD} 4^{+}, \mathrm{CD}^{+}$, or NKT cells by positive selection that is mediated by MHC-I, MHC-II, or CD1d molecules, respectively. The NKT cell developmental pathway starts with positive selection when a DP precursor expresses a correctly rearranged $\alpha \beta T C R$ and interacts with other DP thymocytes expressing a CD1d molecule, presumably presenting self-glycolipids. After positive selection, iNKT cells leave the thymus at an immature stage as defined by the absence of NK receptor expression, for example, NK1.1, and undergo terminal maturation in the periphery. ${ }^{16}$ For iNKT as well as mNKT cells, it is currently unknown what signals regulate this final differentiation.

Several proteins and transcription factors have been shown to be essential for the development of murine iNKT cells, including the adaptor SLAM-associated protein (SAP), calcineurin, and the transcription factors c-Myc, early growth factor 2 (Egr2), and promyelocytic leukemia zinc-finger (PLZF) ${ }^{17-21}$ Furthermore, iNKT cell differentiation has recently been shown to be regulated by transforming growth factor (TGF- $\beta$ ) and Wiskott-Aldrich syndrome protein signaling. ${ }^{22,23}$

It can be noted that $\mathrm{mNKT}$ cells were detected in normal numbers and phenotype in the blood of five SAP-deficient patients, ${ }^{24}$ suggesting that SAP-mediated signal transduction is not involved in human mNKT cell development.

The role of PLZF in mNKT cell development is more complicated as PLZF is not expressed in murine mNKT cells, but can be found in high levels in human blood mNKT cells. ${ }^{24}$ This could be explained by the fact that PLZF was found to induce a transcriptional program in mice that leads to the generation of $\mathrm{CD} 4{ }^{+} \mathrm{T}$ cells with a memory phenotype ${ }^{25}$ corresponding with the naive phenotype of murine mNKT cells and the antigen-experienced phenotype of human mNKT cells in peripheral blood. ${ }^{24}$

These data suggest that differentiation of the various NKT cell subsets is regulated by distinct mechanisms and emphasizes discrepancies between murine and human NKT cell differentiation. Future studies are necessary to delineate the role of these factors for the differentiation and development of human NKT cell populations as well as murine NKT cell populations, other than iNKT cells.

\section{NKT cell location}

Several reports indicate the presence of different NKT cell populations in the intestines of both mice and humans. However, in part due to the inconsistent use of definitions for NKT cells, there is a great variation among the different studies regarding the exact numbers of these cells. Furthermore, conventional T cells have been found to upregulate NK markers upon activation such as NK1.1, making the distinction between NKT and activated conventional $\mathrm{T}$ cells even more complicated. ${ }^{6}$ 
In mice, NKT cells are found at the highest frequency in the liver (10-40\% of liver lymphocytes) and in lower frequencies $(<1 \%)$ in the thymus, bone marrow, spleen, lymph nodes, and blood. ${ }^{26}$ In the murine intestine, $4-10 \%$ of the IELs within small or large intestine and LP lymphocytes (LPLs) were shown to co-express TCR-CD3 $\varepsilon$ complexes and NK cell receptors. ${ }^{27,28}$ Most of these cells were $\mathrm{CD} 8 \alpha^{+}$and a large proportion of these cells were identified as $\gamma \delta \mathrm{T}$ cells. ${ }^{28}$ It can be noted that CD3\&/ $\mathrm{NK} 1.1^{+}$cells may still be present within the intestinal tissues in the absence of CD1d. ${ }^{27,28}$

In humans, NKT cells were found in the same organs; however, the calculated frequencies were lower compared with mice. ${ }^{26}$ In the human intestine, it was estimated that $<0.4 \%$ of all T cells were iNKT cells and are mainly localized within the LP based on expression of the invariant TCR. $\left({ }^{12,29,30}\right.$ and reviewed in Wingender and Kronenberg ${ }^{3}$ )

With the development of tetrameric complexes of $\alpha$ GalCer-loaded CD1d molecules, iNKT cells are relatively easily studied in mice and humans. ${ }^{7,8}$ To date, however, the physiological localization of NKT cell subsets within tissues is still largely unclear due to the lack of antibodies that work efficiently for immunohistochemical analysis of intestinal tissue.

It has been established that mNKT cells in both humans and mice primarily accumulate in the intestinal LP. Furthermore, human mNKT cells represent up to $1-4 \%$ of total peripheral blood T cells, a much higher number compared with the reported frequency of $\sim 0.2 \%$ of human iNKT cells in peripheral blood. ${ }^{24,31}$ Presumably, the newly synthesized anti-V 27.2 monoclonal antibody will provide more detailed information on the location and frequency of human mNKT cells. ${ }^{24}$

\section{Intestinal mNKT cells}

Similar to iNKT cells, mNKT cells are characterized by the expression of a semi-invariant TCR, consisting of V $\alpha 7.2-\mathrm{J} \alpha 33$ in humans and V $\alpha 19-J \alpha 33$ in mice, combined with a limited number of $\mathrm{V} \beta$-chains $(\mathrm{V} \beta 2$ and $\mathrm{V} \beta 13$ in humans, and $\mathrm{V} \beta 6$ and V $\beta 8$ in mice). These TCRs recognize glycolipids presented by the MHC-related molecule 1 or MR1, an MHC class I-like molecule that is highly conserved in mammals. ${ }^{9}$ At present, the nature of the antigens presented by MR1 remains unknown. ${ }^{32}$ The development of mNKT cells is dependent on the presence of commensal microbiota and on the expression of MR1 by bone marrow-derived cells. As mNKT cells were found to specifically accumulate in the LP of the intestine, this suggests that these cells may, in fact, be directed to microbial antigens presented by MR1 molecules. ${ }^{9}$

mNKT cells have been detected in the human thymus and in mouse fetal thymic organ cultures. ${ }^{24}$ As these cells were absent in nude mice, it is likely that mNKT cells develop in the thymus, similar to other NKT cell subsets and conventional T cells. ${ }^{33}$ Furthermore, it was found in mice that B cells are not required for thymic development of mNKT cells, but can however promote the expansion of these cells in the periphery. Interestingly, MR1-deficient B cells were found to be almost as efficient in inducing mNKT cell expansion as MR1-proficient cells, suggesting that cognate interactions between the invariant TCR and MR1 might not be necessary for this expansion. ${ }^{24}$

In mice, as well as in human cord blood, mNKT cells show a naive phenotype, as they lack expression of antigen-experienced markers, such as NK1.1, CD25, CD69, or ICOS. ${ }^{24}$ In contrast, mTCR transgenic mice ${ }^{34,35}$ and human adult mNKT cells express markers that are associated with a memory phenotype. ${ }^{24}$ Finally, murine mNKT cells have been shown to express the chemokine receptor CXCR6 and integrin $\alpha 4 \beta 7$, which are required for gut homing. ${ }^{24}$

\section{Intestinal $\gamma \delta$ NKT cells}

The $\gamma \delta$ T cells are a highly conserved subset of T cells in mice, which constitute $1-5 \%$ of the blood or peripheral organ lymphocytes, but can account for up to $50 \%$ of the lymphocytes that are present within the mucosal epithelia. ${ }^{36}$ These cells express memory cell-surface markers and are capable of rapid secretion of effector cytokines.

Recently, it was found that murine $\gamma \delta \mathrm{T}$ cell differentiation and effector functions are regulated by the TCR signaling molecule Itk, a Tec family tyrosine kinase. ${ }^{37}$ Itk has been shown earlier to suppress the development of innate $\alpha \beta T$ cells and to promote the development of conventional $\alpha \beta T$ cells. $^{38}$ Interestingly, Itk ${ }^{-1-}$ mice harbor increased numbers of $\mathrm{CD} 4^{+} \mathrm{NK} 1.1^{+} \gamma \delta \mathrm{T}$ cells, known as $\gamma \delta \mathrm{NKT}$ cells, which also express PLZF, the transcription factor essential for $\alpha \beta \mathrm{NKT}$

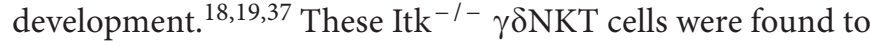
produce high levels of IL-4, IL-10, and IL-13 in addition to IFN- $\gamma$ and expressed B-cell co-stimulatory molecules, such as ICOS, CD40L, and OX40, suggesting a role for these cells in Bcell activation and IgE class switching. ${ }^{37}$ These findings suggest that Itk regulates the lineage development and effector function of murine $\gamma \delta \mathrm{NKT}$ cells.

In the murine intestine, it was found that upon induction of colitis with dextran sulfate sodium, colonic IELs that bear $\gamma \delta$ TCRs $(\gamma \delta$ IEL $)$ induce a transcriptional program that includes protection of epithelial cells, antibacterial defense, and inflammatory cell recruitment. ${ }^{39}$ These $\gamma \delta$ IELs were found to be essential for controlling bacterial penetration across injured mucosal surfaces. Intestinal microbes were required for enhanced expression of both the antibacterial factor RegIII $\gamma$ and several chemotactic cytokines that function in inflammatory cell recruitment. Moreover, the $\gamma \delta$ IEL injury responses that were abrogated in germ-free mice could be restored with the conventional microbiota, suggesting that $\gamma \delta$ IELs need an appropriate bacterial signal to respond. ${ }^{39}$ In another study, it was shown that $\gamma \delta \mathrm{T}$ cells can act as regulatory cells that are anergic in vitro, but can suppress conventional T-cell proliferation and ameliorate 2,4,6trinitrobenzene sulphonic acid (TNBS)-induced colitis in vivo by the production of TGF- $\beta$ and IL-10. ${ }^{40}$ These findings provide new insights into the role of $\gamma \delta$ T cells in maintaining intestinal tissue homeostasis.

As $\gamma \delta \mathrm{NKT}$ cells are part of the $\gamma \delta \mathrm{T}$ cell subset, and not all studies have included the analysis of NKT cell markers, findings on the role of $\gamma \delta$ T cells in mucosal immunity may also implicate a role for $\gamma \delta$ NKT cells. 


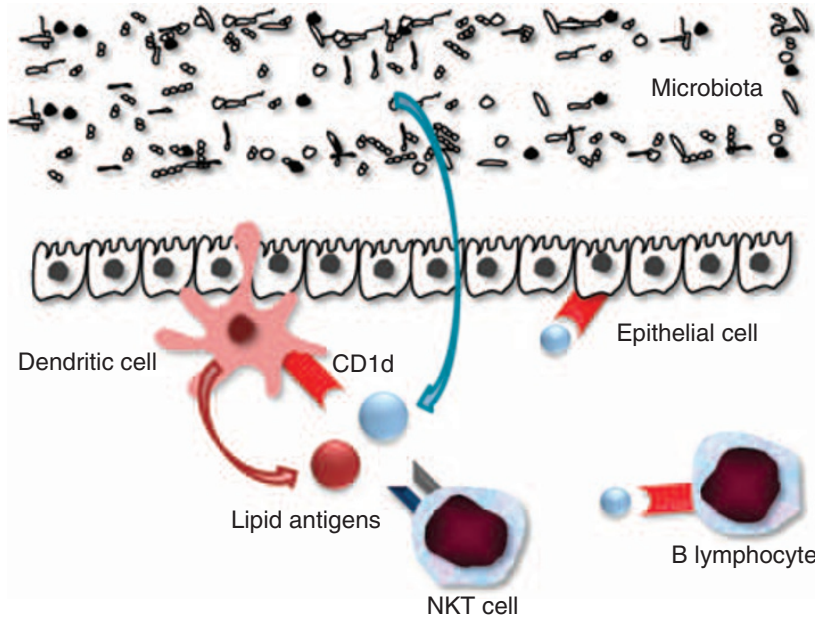

Figure 1 Various cell types within the intestinal mucosa, such as the intestinal epithelial cells (IEC), dendritic cells (DC), and B lymphocytes functionally express the antigen-presenting molecule CD1d.

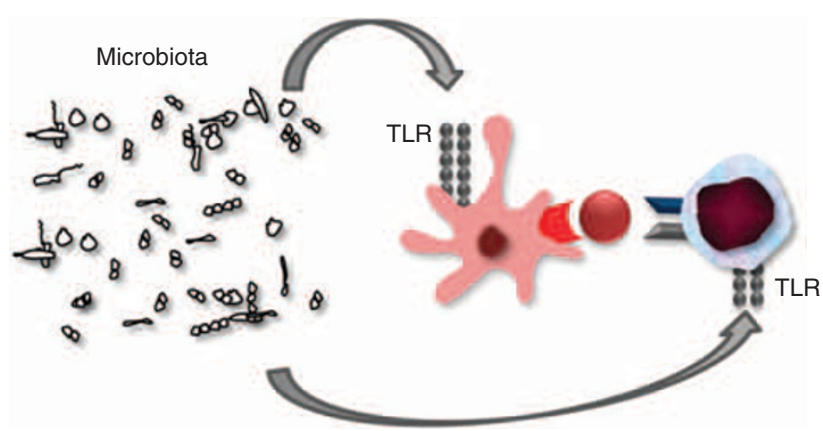

Figure 2 Direct or indirect activation of natural killer T cells (NKT) cells through Toll-like receptor (TLR)-mediated activation of NKT cells or dendritic cells, respectively.

\section{CD1d expression in the intestine}

$\mathrm{CD} 1 \mathrm{~d}$ is a MHC class I-related molecule that consists of a light chain ( $\beta 2$-microglobulin) that is non-covalently associated with a heavy chain. ${ }^{41}$ Furthermore, CD1d is structurally related to HLA-A, HLA-B, and HLA-C proteins. ${ }^{5}$ CD1d is expressed by professional APCs, such as dendritic cells, macrophages, and B cells, and non-professional APCs such as hepatocytes and intestinal epithelial cells (IECs) (Figure 1 and refs. 42-44). CD1d molecules are essentially non-polymorphic, suggesting that these molecules can present only a limited set of (glycolipid) antigens. There is a high degree of conservation of the canonical TCRs and CD1d molecules that allow interspecies cross-reactivity, indicating that these cells have a central role in immunity.

IECs have been found to be capable of presenting antigen to both conventional and NKT cells within the pool of intestinal lymphocytes. Moreover, human IEC were found to be able to activate NKT cells in vitro in a CD1d-dependent manner, ${ }^{45,46}$ suggesting that $\mathrm{CD} 1 \mathrm{~d}$ expression by intestinal cells may serve as a target for NKT cells, contributing to the chronic phase of intestinal inflammation (Figure 1 and refs. 47,48).
Next to a role in antigen presentation, CD1d was also shown to be capable of cellular activation in the absence of NKT cells. In particular, ligation of $\mathrm{CD} 1 \mathrm{~d}$ on IECs gave rise to retrograde epithelial signaling and IL-10 production. ${ }^{49}$ The latter mechanism may thus contribute to tissue homeostasis and control of inflammation at the mucosal surfaces.

\section{NKT cell ligands}

The knowledge on the nature of ligands presented by MR1restricted or CD1d-restricted NKT cells in vivo remains limited. ${ }^{50}$ The most efficient compound that activates classical iNKT cells is a synthetic glycolipid, originally derived from a marine sponge, known as $\alpha \mathrm{GalCer}{ }^{51}$ This molecule binds efficiently to CD1d, and the CD1d: $\alpha$ GalCer complex binds the iNKT TCR, ${ }^{51,52}$ leading to the activation of these cells in both mice and humans. ${ }^{53}$ Several studies have shown that $\alpha$ GalCer is a very potent NKT cell agonist. ${ }^{51,52}$ It has been shown that iNKT cells can respond to glycosphingolipids derived from the cell walls of Gram-negative Sphingomonas spp., ${ }^{54-56} \alpha$-galactosyl diacylglycerols from Borrelia burgdorferi, ${ }^{57}$ and phosphatidylinositol mannoside from mycobacteria. ${ }^{58}$ Recently, the endogenous lysosomal lipid isoglobotrihexosylceramide was shown to potently activate both mouse and human NKT cells, and was proposed as an endogenous ligand for iNKT cells. ${ }^{59}$ To date, these findings have found insufficient support ${ }^{60,61}$ that leaves open the quest for the endogenous antigens for iNKT cells. ${ }^{62}$

The activation of human APCs by Toll-like receptor (TLR) ligands modulates the lipid biosynthetic pathway, resulting in enhanced recognition of CD1d-associated lipids by NKT cells and increased IFN- $\gamma$ production. ${ }^{63}$ Together with the identification of functional TLRs on NKT cells, ${ }^{64}$ these results suggest that NKT cells may be regarded as key players in microbial sensing and the orchestration of ensuing mucosal immune responses (Figure 2).

mNKT cells have been shown to be stimulated by the glycolipid $\alpha$-mannosylceramide ( $\alpha$ ManCer) ${ }^{65}$ To date, these findings have not been confirmed and the structure of MR1 does not appear to be suitable for lipid binding. ${ }^{66}$ Intriguingly, $\alpha$ ManCer has been used as a negative lipid control for iNKT activation by $\alpha \mathrm{GalCer}^{67,68}$ or even as a CD1d glycolipid antagonist. ${ }^{69}$

Specific ligands for $\gamma \delta$ NKT have not been described yet.

\section{NKT cell effector functions}

NKT cells can mediate potent immune regulatory functions in autoimmunity, cancer, infection, and tolerance. ${ }^{70}$ The biological function of NKT cells is paradoxical, because these cells can rapidly produce large amounts of T-helper type 1 (Th1), Th2, and regulatory cytokines. NKT cells may thus promote or suppress cell-mediated immunity in different conditions. ${ }^{70}$

It has been suggested that these diverse functions of NKT cells are regulated by functionally distinct NKT cell subsets, broadly divided into $\mathrm{CD}^{+}{ }^{+}$and $\mathrm{CD} 4{ }^{-}$NKT cells. ${ }^{6}$ Mouse $\mathrm{CD} 4^{+}$and $\mathrm{CD} 4^{-}$NKT cells are distinct in their ability to regulate immune responses in vivo. However, there is no clear distinction in their ability to produce different cytokines. ${ }^{71}$ Mouse NKT cells have been recognized as immunosuppressive cells that produce Th2 

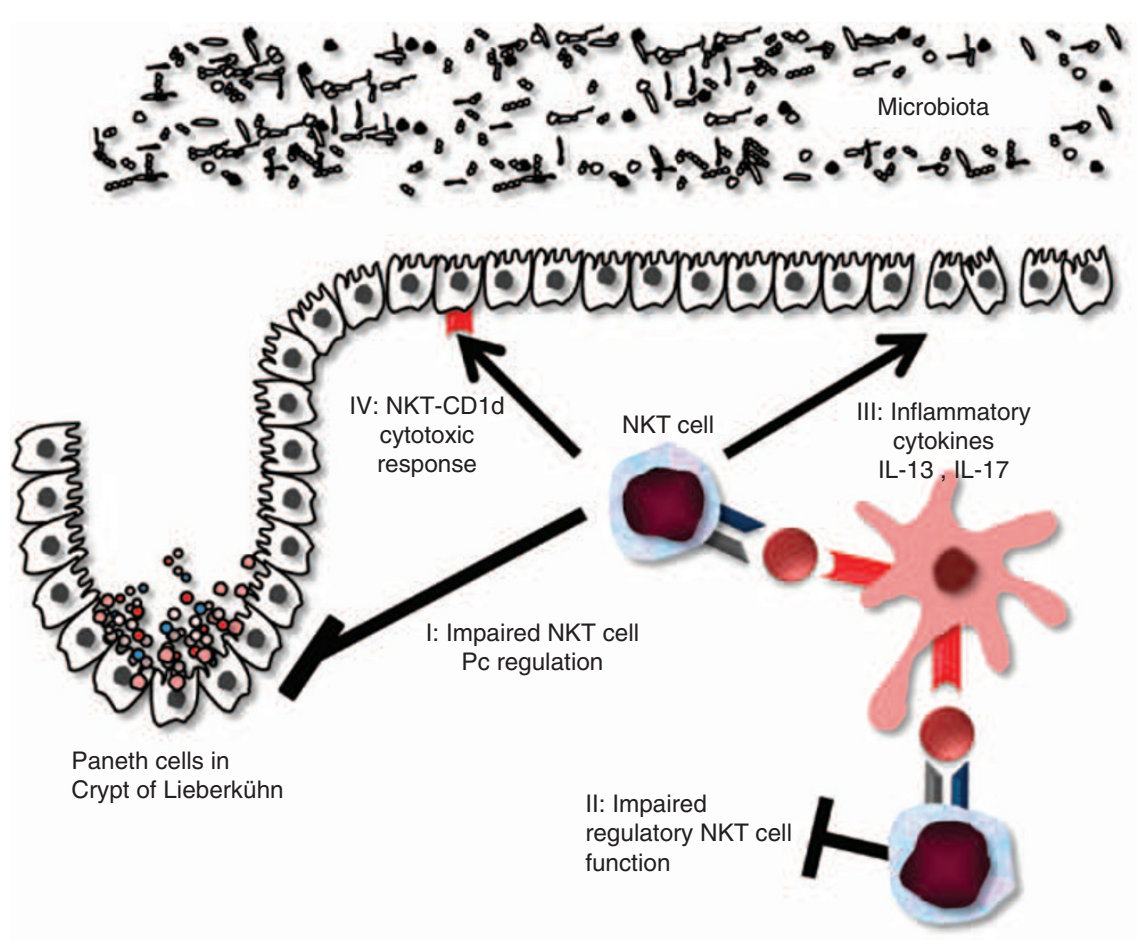

Figure 3 Four mechanisms for the role of natural killer T cells (NKT cells) in intestinal inflammation. I: Impaired NKT cell function leads to decreased production of antimicrobial proteins and subsequently to enhanced local microbial colonization that is associated with intestinal inflammatory disease. II: Absence or inhibition of mucosal regulatory NKT cells (e.g., by producing interleukin (IL)-22) disrupts homeostasis through unopposed mucosal T-cell effector responses. III: Overt production of deleterious cytokines, such as IL-13 and IL-17, by NKT cells disrupts epithelial cell integrity. IV: Activated cytotoxic NKT cells target the CD1d-expressing epithelial barrier.

cytokines or IL-10, whereas in other conditions these cells appear to promote enhanced cell-mediated immunity through the production of Th1 cytokines. ${ }^{72,73}$

It was shown that human $\mathrm{CD} 4^{+} \mathrm{NKT}$ cells can produce both Th1 and Th2 cytokines, whereas CD4- NKT cells primarily produce only Th 1 cytokines. ${ }^{74}$ In the human colon and duodenum, most of the NKT cells are CD161 ${ }^{+}$, and these cells account for about half of all $\mathrm{T}$ cells in both the epithelial layer and the LP. Almost all of the CD161 ${ }^{+} \mathrm{T}$ cells express an $\alpha \beta T C R$ and are able to express both $\mathrm{CD} 4^{+}$or $\mathrm{CD} 8^{+}$cells, but the majority of the cells within the IEL compartment is $\mathrm{CD}^{+}$, whereas $\mathrm{CD} 4^{+}$ cells predominate the LPL population..$^{30}$

Regarding the function of mNKT cells, many questions are still unanswered. So far, murine mNKT cells have been shown to rapidly produce effector cytokines, including IL-4, IL-5, IL$10, \mathrm{TNF} \alpha$, and IFN $-\gamma$ upon activation through MR1. ${ }^{34,35}$ The mNKT cell-specific anti-V $\alpha 7.2$ antibody will enable further functional analysis of these cells in humans. ${ }^{24}$

\section{IL17- and IL22-producing NKT cells in the intestine}

It has been shown that, similar to Th17 cells, ${ }^{75}$ NKT cells can express the pro-inflammatory cytokines IL-17 and IL-22. ${ }^{76,77}$ The IL17-producing NKT cells were NK1.1 ${ }^{-} \mathrm{DX} 5^{+} \mathrm{TCR} \beta^{+}$ and shown to constitutively express IL-23R and the ROR $\gamma \mathrm{t}$ transcription factor. ${ }^{78,79}$ The production of IL-17 by NKT cells may have an important role in the induction of IBD, as increased expression of IL-17 has been reported in both patients with $\mathrm{IBD}^{80,81}$ and animal models of this disease (Figure 3 and refs. 82,83). In addition, it was found that polymorphisms in IL-17A and IL-17F genes are associated with increased susceptibility to UC. ${ }^{84}$

IL-22, a cytokine of the IL-10 family, is one of the key cytokines that mediates the crosstalk between epithelial cells and immune cells. IL-22 is produced by various leukocytes, including $\mathrm{CD} 4^{+}$and $\mathrm{CD} 8^{+} \mathrm{T}$ cells, $\gamma \delta \mathrm{T}$ cells, NK cells, and myeloid cells. ${ }^{85,86}$ Recently, IL-22 has also been shown to be produced by Th17, NK, and, as mentioned, by NKT cells. ${ }^{75,76,87,88}$ IL-22 is implicated in the restoration of barrier function upon epithelial damage during inflammation. Furthermore, IL-22 has been found to enhance the production of antimicrobial peptides, proinflammatory cytokines, and chemokines by epithelial cells. ${ }^{89-91}$

The production of IL-17 or IL-22 by subsets of NKT cells may have an important role in physiological processes, such as microbial defense as well as pathological immune responses, which are associated with IBD (Figure 3 and ref. 92).

\section{NKT CELLS IN DISEASE}

\section{Oral tolerance}

In the intestine, DCs continuously take up and transport antigens from the gut lumen to draining lymph nodes, in which they interact with antigen-specific $T$ cells. Under homeostatic conditions, this interaction will result in the unresponsiveness of T cells, thus providing one of the mechanisms for (oral) tolerance 
toward harmless environmental antigens. Specific activation by additional signals during the interaction of DCs and T cells can block T-cell tolerance induction and instead give rise to inflammatory T-cell responses. ${ }^{93}$

NKT cell-deficient mice were shown to have an impaired tolerance induction, suggesting that NKT cells have a critical role in oral tolerance. ${ }^{94}$ In these knockout mice, treatment with high- and low-dose ovalbumin failed to induce IL-10 and TGF $\beta$-producing $\mathrm{CD} 25^{+} \mathrm{CD} 4{ }^{+}$regulatory $\mathrm{T}$ cells $\left(\mathrm{T}_{\text {reg }}\right.$ cells) in Peyer's patches and in the spleen. ${ }^{94}$ Accordingly, in the presence of NKT cells, DCs were found to produce more IL-10 and lose the ability to produce IL-12, consistent with a tolerogenic DC function..$^{95}$ As such, it was proposed that activated NKT cells contribute to the induction of tolerogenic DCs. Furthermore, it was shown that IL-4 and IL-10-producing $\mathrm{CD} 4{ }^{+}$iNKT cells are required for the induction of oral nickel tolerance and for the infectious spread of tolerance from APCs to $\mathrm{T}_{\text {reg }}$ cells. ${ }^{96}$

In contrast to the proposed role for NKT cells in tolerance induction, some studies have shown that NKT cells are not required for induction of oral tolerance. ${ }^{97}$ Injection of $\alpha$ GalCer was shown to activate NKT cells and in turn trigger maturation of splenic DC, thereby blocking the induction of oral tolerance. ${ }^{98}$ Along these lines, mucosal administration of $\alpha$ GalCer has been proposed as a mucosal adjuvant in two different vaccination studies. ${ }^{99,100}$ Although $\alpha \mathrm{GalCer}$ may serve as an artificial ligand for mucosal vaccination, the activation of NKT cells by $\alpha$ GalCer in vivo may not reflect physiological activation of NKT cells that contributes to tolerance induction.

In conclusion, the role for NKT cells in tolerance induction is still controversial and the outcome may depend on the experimental models applied. ${ }^{101,102}$

\section{NKT cells in IBD}

Both IECs and DCs functionally express CD1d on the cell surfaces. ${ }^{5,46}$ Oxazolone colitis, a mouse model of human UC, was found to be associated with CD1d-dependent production of Th2 cytokines involved in mucosal inflammation. ${ }^{67,103}$ In particular, CD1d-deficient mice, iNKT cell-deficient mice, and wild type (WT) mice treated with CD1d-blocking antibodies were protected from the development of this colitis. Treatment with an IL-13-decoy receptor prevented tissue inflammation upon intestinal oxazolone exposure, thereby identifying IL13 as a functionally important and deleterious cytokine in this pathway. ${ }^{103,104}$ This finding correlates with patients with UC, who exhibited increased Th2 cytokine secretion by LP mononuclear cells upon CD1d-NKT cell stimulation. ${ }^{12}$ Interestingly, earlier studies in another mouse colitis model suggested that CD1d-restricted NKT cell activation with $\alpha$ GalCer could protect from colitis. ${ }^{105,106}$ Therefore, mucosal NKT cells may be involved in both protective and pathogenic immunity. Whether these distinct NKT cell effector functions in colitis are imprinted depending on the specificity of the lipid antigen or whether these NKT cells are regulated within the intestinal tissue remains to be solved. ${ }^{6,107}$

\section{NKT cell-DC interactions in the small intestine LP}

Several distinct subsets of LP-DCs have been described, ${ }^{102,108,109}$ of which the $\mathrm{CD} 103^{+} \mathrm{CD} 11 \mathrm{~b}^{+} \mathrm{CD} 8 \alpha^{+}$subset is able to migrate to the mesenteric lymph node (MLN). ${ }^{110,111}$ It was shown that LP-DCs continuously migrate from the small intestine LP to the MLN in a CCR7-dependent manner. ${ }^{10,112}$ The finding that CCR7 expression by LP-DCs was increased upon $\alpha$ GalCer treatment, indicates that NKT activation can promote LP-DCs migration to the MLN. ${ }^{101}$ Next to a role in regulation of local DC recruitment, NKT cells can further activate DCs through the production of cytokines such as IFN- $\gamma{ }^{113,114}$

It has become clear that different mucosal subsets of DCs differentially express a range of microbial pattern recognition molecules. ${ }^{115}$ There is abundant evidence that iNKT cells can be activated by microbial products such as lipopolysaccharide $55,64,116$ during antigen presentation. Several findings suggested a positive feedback loop, where lipopolysaccharide activates TLR4-expressing APCs to produce IL-12 and IL-18, which in turn induces early IFN $-\gamma$ production by iNKT cells. This IFN- $\gamma$ feeds back to the APCs to potentiate TNF $\alpha$ production, leading to further APC activation. ${ }^{113}$

Alternatively, iNKT cells have been reported to express TLR4 and can thus be directly activated by lipopolysaccharide. ${ }^{64}$ TLR or CD14 expression by NKT cells, however, is still debated and therefore needs further confirmation. ${ }^{113}$

In summary, some bacteria can activate NKT cells either directly through microbial glycolipid antigens ${ }^{2,54,117}$ or indirectly through TLR signaling by APCs and perhaps also NKT cells (Figure 2). Interactions between DCs, NKT cells, and bacteria may therefore crucially contribute to both physiological and pathological immune responses within the intestinal tissue.

\section{NKT cell-B cell interactions in the small intestine}

CD1d is detectable on nearly all B cells, and cognate CD1ddependent interaction with NKT cells can elicit B-cell proliferation and antibody production. ${ }^{118,119}$ Earlier studies showed that chronic intestinal inflammation induces the upregulation of CD1d expression on B cells in the MLN and LP, leading to enhanced IL-10 production that can suppress the progression of intestinal inflammation. ${ }^{120}$ These studies identified a specific regulatory role for $\mathrm{CD} 1 \mathrm{~d}$-expressing $\mathrm{B}$ cells within the intestinal tissues.

A recent study showed that conventional IEL preparations contain a specific subset of $B$ cells that resides in the superficial LP (SLP) of the small and large intestine. ${ }^{121}$ The formation of SLP B cells in the small intestinal villi was completely dependent on lymphotoxin- $\alpha$ and, partially, on proper BCR signaling. Interestingly, these B cells were not dependent on the resident microbiota or innate microbial sensing, but were specifically reduced in the small intestine of iNKT cell-deficient mice. As it was found that SLP B cells and iNKT cells are fully preserved in germ-free mice, ${ }^{121,122} \mathrm{~B}$ cell-NKT cell interaction may be dependent on food antigens or endogenous glycolipids, rather than on commensal microbial antigens. However, additional studies are required to understand the relationship between this special subset of $\mathrm{B}$ cells in the small intestinal villi and NKT cells. 


\section{Regulation of bacterial colonization by CD1d-restricted NKT cells}

CD1d-restricted NKT cells have been shown to be involved in the host defense against various bacterial, fungal, and viral pathogens, including those that reside in the lung and intestine. ${ }^{5,67}$ Although the exact ligands involved in this antimicrobial NKT cell activation are not yet identified, they may include both pathogenic and host-derived glycolipids that are induced by the microbiota. ${ }^{54,55,59}$

Most of the bacteria residing within the intestinal lumen are located in the colon, whereas colonization of the small intestine remains limited, suggesting the existence of potent local regulatory mechanisms. The current understanding of how bacterial commensalism is established in mammals and which host factors contribute to this process is relatively unknown. ${ }^{123}$ Bacteria can interact with the host at a cellular level through contact with IEC and hematopoietic cells, which are associated with innate and adaptive immune responses. ${ }^{124}$ For example, $B$ cells can respond to bacterial products by the production of bacteria-specific antibodies (mainly IgA), thus disabling bacterial translocation across the epithelial cell layers. ${ }^{124,125}$ IECs can regulate homeostasis by responding to bacterial-derived antigens through the expression and activation of microbial pattern recognition molecules such as TLRs. ${ }^{126}$

Within the small intestine, Paneth cells (Pcs) are located at the bottom of the crypts of Lieberkühn. Upon activation, Pcs secrete various antibacterial proteins, such as cryptdins or $\alpha$-defensins, into the intestinal crypts. ${ }^{127}$ As Pcs are located mainly in the small intestine, their presence may contribute to the relative low grade of bacterial colonization at that specific segment of the intestinal tract. ${ }^{128,129}$ Previously, Pcs in mice were found to express CD1d, ${ }^{130}$ similar to the CD1d expression on IECs ${ }^{43,46,131,132}$ and hematopoietic cells. ${ }^{133}$ We have recently shown that colonization of the intestine with a range of bacteria (Pseudomonas aeruginosa, Staphylococcus aureus, non-pathogenic Escherichia coli, and Lactobacillus gasseri) is regulated by CD1d. ${ }^{68} \mathrm{CD} 1 \mathrm{~d}$-deficient mice were found to have an altered bacterial composition including increased numbers of adherent bacteria. As a mechanism of action, CD1d-mediated pathways were shown to regulate Pc function. In vitro studies indicated that CD1d expression by IEC and/or Pc was sufficient for NKT cell-mediated Pc granule content release. In addition, other mucosal CD1d-expressing APCs may be involved.

It has been proposed that intestinal NKT cells may regulate Pc function upon recognition of bacteria-derived lipid antigens (Figure 3), and thus take part in reciprocal interactions between the microbiota and host cells. On the basis of various bacteria that are seemingly regulated by CD1d-restricted mechanisms, it seems more likely that colonization induces a common (endogenous) antigen that is responsible for local NKT activation. ${ }^{68}$ This CD1d-restricted pathway may represent an alternative to the previously described TLR-induced Pc activation routes. ${ }^{127}$ As such, NKT cells have now been implicated in at least three mechanisms of antimicrobial defense. First, through NKT cell expression and release of antimicrobial mediators by cells, for example, granulysin; ${ }^{134}$ second, through activation of innate (e.g., macrophages ${ }^{67}$ ) and adaptive immune cells involved in antimicrobial responses; ${ }^{135}$ and third, through the regulation of non-myeloid cells such as Pc. ${ }^{68}$

Given the function of $\mathrm{Pc}$ in the regulation of mucosal homeostasis, we hypothesize that aberrant CD1d-NKT cell pathways may be involved in the pathogenesis of IBD. Along similar lines, it has been shown that polymorphisms within the genes NOD2/CARD15, ATG16L1, or XBP-1 are associated with IBD. ${ }^{136-139}$ In all of these cases, the described mutations lead to Pc dysfunction and impaired microbial clearance. It has been suggested that in these conditions, overt microbial translocation will give rise to excessive mucosal immune responses that are key to the IBD pathogenesis. Similarly, inadequate or dysregulated CD1d-restricted NKT cell regulation of Pc could contribute to the development of entero-colitis (Figure 3 and ref. 68).

\section{CONCLUDING REMARKS}

Currently, many studies focus on the discovery of novel NKT cell ligands from microorganisms and antigens that are derived from endogenous sources. Next to the thoroughly studied classical CD1d-restricted iNKT cells, novel (invariant) T-cell subsets with other selecting antigen-presenting molecules have been discovered. This emerging knowledge will contribute to our understanding of the complex interplay between the microbiota and immune cells in homeostatic and inflammatory conditions of the intestinal mucosa.

\section{REFERENCES}

1. Bendelac, A., Savage, P.B. \& Teyton, L. The biology of NKT cells. Annu. Rev. Immunol. 25, 297-336 (2007).

2. van Dieren, J.M. et al. Roles of CD1d-restricted NKT cells in the intestine. Inflamm. Bowel Dis. 13, 1146-1152 (2007).

3. Wingender, G. \& Kronenberg, M. Role of NKT cells in the digestive system. IV. The role of canonical natural killer T cells in mucosal immunity and inflammation. Am. J. Physiol. Gastrointest. Liver Physiol. 294, G1-8 (2008).

4. Zeissig, S., Kaser, A., Dougan, S.K., Nieuwenhuis, E.E. \& Blumberg, R.S. Role of NKT cells in the digestive system. III. Role of NKT cells in intestinal immunity. Am. J. Physiol. Gastrointest. Liver Physiol. 293, G1101-1105 (2007).

5. Brigl, M. \& Brenner, M.B. CD1: antigen presentation and T cell function. Annu. Rev. Immunol. 22, 817-890 (2004).

6. Godfrey, D.I., MacDonald, H.R., Kronenberg, M., Smyth, M.J. \& Van Kaer, L. NKT cells: what's in a name? Nat. Rev. Immunol. 4, 231-237 (2004).

7. Benlagha, K., Weiss, A., Beavis, A., Teyton, L. \& Bendelac, A. In vivo identification of glycolipid antigen-specific T cells using fluorescent CD1d tetramers. J. Exp. Med. 191, 1895-1903 (2000).

8. Matsuda, J.L. et al. Tracking the response of natural killer T cells to a glycolipid antigen using CD1d tetramers. J. Exp. Med. 192, 741-754 (2000).

9. Treiner, E. et al. Selection of evolutionarily conserved mucosalassociated invariant T cells by MR1. Nature 422, 164-169 (2003).

10. Chiu, Y.H. et al. Distinct subsets of CD1d-restricted T cells recognize self-antigens loaded in different cellular compartments. J. Exp. Med. 189, 103-110 (1999).

11. Jahng, A. et al. Prevention of autoimmunity by targeting a distinct, noninvariant CD1d-reactive T cell population reactive to sulfatide. J. Exp. Med. 199, 947-957 (2004).

12. Fuss, I.J. et al. Nonclassical CD1d-restricted NK T cells that produce IL-13 characterize an atypical Th2 response in ulcerative colitis. J. Clin. Invest. 113, 1490-1497 (2004). 
13. Arrenberg, P., Halder, R. \& Kumar, V. Cross-regulation between distinct natural killer $T$ cell subsets influences immune response to self and foreign antigens. J. Cell. Physiol. 218, 246-250 (2009).

14. Halder, R.C., Aguilera, C., Maricic, I. \& Kumar, V. Type II NKT cellmediated anergy induction in type I NKT cells prevents inflammatory liver disease. J. Clin. Invest. 117, 2302-2312 (2007).

15. Lees, R.K., Ferrero, I. \& MacDonald, H.R. Tissue-specific segregation of TCRgamma delta+ NKT cells according to phenotype TCR repertoire and activation status: parallels with TCR alphabeta+NKT cells. Eur. J. Immunol. 31, 2901-2909 (2001).

16. Matsuda, J.L., Mallevaey, T., Scott-Browne, J. \& Gapin, L. CD1drestricted iNKT cells, the "Swiss-Army knife" of the immune system. Curr. Opin. Immunol. 20, 358-368 (2008).

17. Griewank, K. et al. Homotypic interactions mediated by Slamf1 and Slamf6 receptors control NKT cell lineage development. Immunity 27, 751-762 (2007).

18. Kovalovsky, D. et al. The BTB-zinc finger transcriptional regulator PLZF controls the development of invariant natural killer T cell effector functions. Nat. Immunol. 9, 1055-1064 (2008).

19. Savage, A.K. et al. The transcription factor PLZF directs the effector program of the NKT cell lineage. Immunity 29, 391-403 (2008).

20. Lazarevic, $\mathrm{V}$. et al. The gene encoding early growth response 2 , a target of the transcription factor NFAT, is required for the development and maturation of natural killer T cells. Nat. Immunol. 10, 306-313 (2009).

21. Mycko, M.P. et al. Selective requirement for c-Myc at an early stage of V(alpha)14i NKT cell development. J. Immunol. 182, 4641-4648 (2009).

22. Doisne, J.M. et al. iNKT cell development is orchestrated by different branches of TGF-beta signaling. J. Exp. Med. 206, 1365-1378 (2009).

23. Astrakhan, A., Ochs, H.D. \& Rawlings, D.J. Wiskott-Aldrich syndrome protein is required for homeostasis and function of invariant NKT cells. J. Immunol. 182, 7370-7380 (2009).

24. Martin, E. et al. Stepwise development of MAIT cells in mouse and human. PLoS Biol. 7, e54 (2009).

25. Raberger, J. et al. The transcriptional regulator PLZF induces the development of CD44 high memory phenotype T cells. Proc. Natl Acad. Sci. USA 105, 17919-17924 (2008).

26. Godfrey, D.I. \& Berzins, S.P. Control points in NKT-cell development. Nat. Rev. Immunol. 7, 505-518 (2007).

27. Bannai, M. et al. Abundance of unconventional CD8(+) natural killer T cells in the large intestine. Eur. J. Immunol. 31, 3361-3369 (2001).

28. Ishimoto, Y. et al. Age-dependent variation in the proportion and number of intestinal lymphocyte subsets, especially natural killer T cells, doublepositive CD4+ CD8+ cells and B220+ T cells, in mice. Immunology 113, 371-377 (2004).

29. liai, T. et al. CD161+T (NT) cells exist predominantly in human intestinal epithelium as well as in liver. Clin. Exp. Immunol. 129, 92-98 (2002).

30. O'Keeffe, J. et al. Diverse populations of T cells with NK cell receptors accumulate in the human intestine in health and in colorectal cancer. Eur. J. Immunol. 34, 2110-2119 (2004).

31. Lee, P.T. et al. Testing the NKT cell hypothesis of human IDDM pathogenesis. J. Clin. Invest. 110, 793-800 (2002).

32. Hansen, T.H., Huang, S., Arnold, P.L. \& Fremont, D.H. Patterns of nonclassical MHC antigen presentation. Nat. Immunol. 8, 563-568 (2007)

33. Tilloy, F. et al. An invariant T cell receptor alpha chain defines a novel TAP-independent major histocompatibility complex class Ib-restricted alpha/beta T cell subpopulation in mammals. J. Exp. Med. 189, 1907-1921 (1999).

34. Croxford, J.L., Miyake, S., Huang, Y.Y., Shimamura, M. \& Yamamura, T. Invariant $\mathrm{V}$ (alpha) $19 \mathrm{i} \mathrm{T}$ cells regulate autoimmune inflammation. Nat. Immunol. 7, 987-994 (2006).

35. Kawachi, I., Maldonado, J., Strader, C. \& Gilfillan, S. MR1-restricted V alpha $19 \mathrm{i}$ mucosal-associated invariant $T$ cells are innate $T$ cells in the gut lamina propria that provide a rapid and diverse cytokine response. J. Immunol. 176, 1618-1627 (2006).

36. Carding, S.R. \& Egan, P.J. Gammadelta T cells: functional plasticity and heterogeneity. Nat. Rev. Immunol. 2, 336-345 (2002).

37. Felices, M., Yin, C.C., Kosaka, Y., Kang, J. \& Berg, L.J. Tec kinase Itk in gammadeltaT cells is pivotal for controlling lgE production in vivo. Proc. Natl Acad. Sci. USA 106, 8308-8313 (2009).

38. Berg, L.J. Signalling through TEC kinases regulates conventional versus innate CD8(+) T-cell development. Nat. Rev. Immunol. 7, 479-485 (2007).
39. Ismail, A.S., Behrendt, C.L. \& Hooper, L.V. Reciprocal interactions between commensal bacteria and gamma delta intraepithelial lymphocytes during mucosal injury. J. Immunol. 182, 3047-3054 (2009).

40. Hoffmann, J.C. et al. Gammadelta T lymphocytes: a new type of regulatory T cells suppressing murine 2,4,6-trinitrobenzene sulphonic acid (TNBS)-induced colitis. Int. J. Colorectal Dis. 23, 909-920 (2008).

41. Zeng, Z. et al. Crystal structure of mouse CD1: an MHC-like fold with a large hydrophobic binding groove. Science 277, 339-345 (1997).

42. Balk, S.P. et al. Beta 2-microglobulin-independent MHC class Ib molecule expressed by human intestinal epithelium. Science 265, 259-262 (1994).

43. Bleicher, P.A. et al. Expression of murine CD1 on gastrointestinal epithelium. Science 250, 679-682 (1990).

44. Blumberg, R.S. et al. Expression of a nonpolymorphic MHC class I-like molecule, CD1D, by human intestinal epithelial cells. J. Immunol. 147, 2518-2524 (1991).

45. Panja, A., Blumberg, R.S., Balk, S.P. \& Mayer, L. CD1d is involved in T cell-intestinal epithelial cell interactions. J. Exp. Med. 178, 1115-1119 (1993).

46. van de Wal, Y. et al. Delineation of a CD1d-restricted antigen presentation pathway associated with human and mouse intestinal epithelial cells. Gastroenterology 124, 1420-1431 (2003).

47. Kita, H. et al. Quantitation and phenotypic analysis of natural killer T cells in primary biliary cirrhosis using a human CD1d tetramer. Gastroenterology 123, 1031-1043 (2002).

48. Metelitsa, L.S., Weinberg, K.I., Emanuel, P.D. \& Seeger, R.C. Expression of CD1d by myelomonocytic leukemias provides a target for cytotoxic NKT cells. Leukemia 17, 1068-1077 (2003).

49. Colgan, S.P., Hershberg, R.M., Furuta, G.T. \& Blumberg, R.S. Ligation of intestinal epithelial CD1d induces bioactive IL-10: critical role of the cytoplasmic tail in autocrine signaling. Proc. Natl Acad. Sci. USA 96, 13938-13943 (1999).

50. Brutkiewicz, R.R. CD1d ligands: the good, the bad, and the ugly. J. Immunol. 177, 769-775 (2006).

51. Kawano, T. et al. CD1d-restricted and TCR-mediated activation of valpha14 NKT cells by glycosylceramides. Science $\mathbf{2 7 8 , 1 6 2 6 - 1 6 2 9}$ (1997).

52. Burdin, N. et al. Selective ability of mouse CD1 to present glycolipids: alpha-galactosylceramide specifically stimulates $\mathrm{V}$ alpha $14+\mathrm{NK} T$ lymphocytes. J. Immunol. 161, 3271-3281 (1998).

53. Sidobre, $\mathrm{S}$. et al. The $\mathrm{V}$ alpha 14 NKT cell TCR exhibits high-affinity binding to a glycolipid/CD1d complex. J. Immunol. 169, 1340-1348 (2002).

54. Kinjo, Y. et al. Recognition of bacterial glycosphingolipids by natural killer T cells. Nature 434, 520-525 (2005).

55. Mattner, J. et al. Exogenous and endogenous glycolipid antigens activate NKT cells during microbial infections. Nature 434, 525-529 (2005).

56. Sriram, V., Du, W., Gervay-Hague, J. \& Brutkiewicz, R.R. Cell wall glycosphingolipids of Sphingomonas paucimobilis are CD1d-specific ligands for NKT cells. Eur. J. Immunol. 35, 1692-1701 (2005).

57. Kinjo, Y. et al. Natural killer T cells recognize diacylglycerol antigens from pathogenic bacteria. Nat. Immunol. 7, 978-986 (2006).

58. Fischer, K. et al. Mycobacterial phosphatidylinositol mannoside is a natural antigen for CD1d-restricted T cells. Proc. Natl Acad. Sci. USA 101, 10685-10690 (2004).

59. Zhou, D. et al. Lysosomal glycosphingolipid recognition by NKT cells. Science 306, 1786-1789 (2004).

60. Porubsky, S. et al. Normal development and function of invariant natural killer T cells in mice with isoglobotrihexosylceramide (iGb3) deficiency. Proc. Natl Acad. Sci. USA 104, 5977-5982 (2007).

61. Speak, A.O. et al. Implications for invariant natural killer T cell ligands due to the restricted presence of isoglobotrihexosylceramide in mammals. Proc. Natl Acad. Sci. USA 104, 5971-5976 (2007).

62. Kronenberg, M. \& Gapin, L. Natural killer T cells: know thyself. Proc. Nat/ Acad. Sci. USA 104, 5713-5714 (2007).

63. Salio, M. et al. Modulation of human natural killer T cell ligands on TLR-mediated antigen-presenting cell activation. Proc. Natl Acad. Sci. USA 104, 20490-20495 (2007).

64. Askenase, P.W. et al. TLR-dependent IL-4 production by invariant Valpha14+Jalpha18+ NKT cells to initiate contact sensitivity in vivo. J. Immunol. 175, 6390-6401 (2005). 
65. Shimamura, M. et al. Modulation of Valpha19 NKT cell immune responses by alpha-mannosyl ceramide derivatives consisting of a series of modified sphingosines. Eur. J. Immunol. 37, 1836-1844 (2007).

66. Huang, S. et al. MR1 uses an endocytic pathway to activate mucosalassociated invariant T cells. J. Exp. Med. 205, 1201-1211 (2008).

67. Nieuwenhuis, E.E. et al. CD1d-dependent macrophage-mediated clearance of Pseudomonas aeruginosa from lung. Nat. Med. 8, 588-593 (2002).

68. Nieuwenhuis, E.E. et al. Cd1d-dependent regulation of bacterial colonization in the intestine of mice. J. Clin. Invest. 119, 1241-1250 (2009).

69. Nieuwenhuis, E.E. et al. CD1d and CD1d-restricted iNKT-cells play a pivotal role in contact hypersensitivity. Exp. Dermatol. 14, 250-258 (2005).

70. Godfrey, D.I. \& Kronenberg, M. Going both ways: immune regulation via CD1d-dependent NKT cells. J. Clin. Invest. 114, 1379-1388 (2004).

71. Crowe, N.Y. et al. Differential antitumor immunity mediated by NKT cell subsets in vivo. J. Exp. Med. 202, 1279-1288 (2005).

72. Smyth, M.J. \& Godfrey, D.I. NKT cells and tumor immunity - a doubleedged sword. Nat. Immunol. 1, 459-460 (2000).

73. Wilson, S.B. \& Delovitch, T.L. Janus-like role of regulatory iNKT cells in autoimmune disease and tumour immunity. Nat. Rev. Immunol. 3, 211-222 (2003).

74. Gumperz, J.E., Miyake, S., Yamamura, T. \& Brenner, M.B. Functionally distinct subsets of CD1d-restricted natural killer $\mathrm{T}$ cells revealed by CD1d tetramer staining. J. Exp. Med. 195, 625-636 (2002).

75. Liang, S.C. et al. Interleukin (IL)-22 and IL-17 are coexpressed by Th17 cells and cooperatively enhance expression of antimicrobial peptides. J. Exp. Med. 203, 2271-2279 (2006).

76. Goto, M. et al. Murine NKT cells produce Th17 cytokine interleukin-22. Cell. Immunol. 254, 81-84 (2009).

77. Michel, M.L. et al. Critical role of ROR-gammat in a new thymic pathway leading to IL-17-producing invariant NKT cell differentiation. Proc. Natl Acad. Sci. USA 105, 19845-19850 (2008).

78. Coquet, J.M. et al. Diverse cytokine production by NKT cell subsets and identification of an IL-17-producing CD4-NK1.1 - NKT cell population. Proc. Natl Acad. Sci. USA 105, 11287-11292 (2008).

79. Rachitskaya, A.V. et al. Cutting edge: NKT cells constitutively express IL-23 receptor and RORgammat and rapidly produce IL-17 upon receptor ligation in an IL-6-independent fashion. J. Immunol. 180, 5167-5171 (2008)

80. Fujino, S. et al. Increased expression of interleukin 17 in inflammatory bowel disease. Gut 52, 65-70 (2003).

81. Nielsen, O.H., Kirman, I., Rudiger, N., Hendel, J. \& Vainer, B. Upregulation of interleukin-12 and -17 in active inflammatory bowel disease. Scand. J. Gastroenterol. 38, 180-185 (2003).

82. Hue, S. et al. Interleukin-23 drives innate and T cell-mediated intestinal inflammation. J. Exp. Med. 203, 2473-2483 (2006).

83. Kullberg, M.C. et al. IL-23 plays a key role in Helicobacter hepaticusinduced T cell-dependent colitis. J. Exp. Med. 203, 2485-2494 (2006).

84. Arisawa, T. et al. The influence of polymorphisms of interleukin-17A and interleukin-17F genes on the susceptibility to ulcerative colitis. J. Clin. Immunol. 28, 44-49 (2008).

85. Dumoutier, L., Louahed, J. \& Renauld, J.C. Cloning and characterization of IL-10-related T cell-derived inducible factor (IL-TIF), a novel cytokine structurally related to IL-10 and inducible by IL-9. J. Immunol. 164, 1814-1819 (2000)

86. Ouyang, W., Kolls, J.K. \& Zheng, Y. The biological functions of T helper 17 cell effector cytokines in inflammation. Immunity 28, 454-467 (2008).

87. Zheng, Y. et al. Interleukin-22, a T(H)17 cytokine, mediates IL-23induced dermal inflammation and acanthosis. Nature 445, 648-651 (2007).

88. Zenewicz, L.A. et al. Innate and adaptive interleukin-22 protects mice from inflammatory bowel disease. Immunity 29, 947-957 (2008).

89. Boniface, K. et al. IL-22 inhibits epidermal differentiation and induces proinflammatory gene expression and migration of human keratinocytes. J. Immunol. 174, 3695-3702 (2005).

90. Sa, S.M. et al. The effects of IL-20 subfamily cytokines on reconstituted human epidermis suggest potential roles in cutaneous innate defense and pathogenic adaptive immunity in psoriasis. J. Immunol. 178, 2229-2240 (2007).

91. Wolk, K. et al. IL-22 regulates the expression of genes responsible for antimicrobial defense, cellular differentiation, and mobility in keratinocytes: a potential role in psoriasis. Eur. J. Immunol. 36, 1309-1323 (2006).

92. Maloy, K.J. The interleukin-23/interleukin-17 axis in intestinal inflammation. J. Intern. Med. 263, 584-590 (2008).

93. Mayer, L. \& Shao, L. Therapeutic potential of oral tolerance. Nat. Rev. Immunol. 4, 407-419 (2004).

94. Kim, H.J., Hwang, S.J., Kim, B.K., Jung, K.C. \& Chung, D.H. NKT cells play critical roles in the induction of oral tolerance by inducing regulatory T cells producing $\mathrm{IL}-10$ and transforming growth factor beta, and by clonally deleting antigen-specific T cells. Immunology 118, 101-111 (2006).

95. Vincent, M.S. et al. CD1-dependent dendritic cell instruction. Nat. Immunol. 3, 1163-1168 (2002).

96. Roelofs-Haarhuis, K., Wu, X. \& Gleichmann, E. Oral tolerance to nickel requires $\mathrm{CD} 4+$ invariant NKT cells for the infectious spread of tolerance and the induction of specific regulatory T cells. J. Immunol. 173, 1043-1050 (2004).

97. Ishimitsu, R., Yajima, T., Nishimura, H., Kawauchi, H. \& Yoshikai, Y. NKT cells are dispensable in the induction of oral tolerance but are indispensable in the abrogation of oral tolerance by prostaglandin $\mathrm{E}$. Eur. J. Immunol. 33, 183-193 (2003).

98. Chung, Y., Chang, W.S., Kim, S. \& Kang, C.Y. NKT cell ligand alphagalactosylceramide blocks the induction of oral tolerance by triggering dendritic cell maturation. Eur. J. Immunol. 34, 2471-2479 (2004).

99. Courtney, A.N. et al. Alpha-galactosylceramide is an effective mucosal adjuvant for repeated intranasal or oral delivery of HIV peptide antigens. Vaccine 27, 3335-3341 (2009).

100. Lindqvist, M., Persson, J., Thorn, K. \& Harandi, A.M. The mucosal adjuvant effect of alpha-galactosylceramide for induction of protective immunity to sexually transmitted viral infection. J. Immunol. 182, 6435-6443 (2009).

101. Chang, J.H. et al. Functional maturation of lamina propria dendritic cells by activation of NKT cells mediates the abrogation of oral tolerance. Eur. J. Immunol. 38, 2727-2739 (2008).

102. Chirdo, F.G., Millington, O.R., Beacock-Sharp, H. \& Mowat, A.M. Immunomodulatory dendritic cells in intestinal lamina propria. Eur. J. Immunol. 35, 1831-1840 (2005).

103. Heller, F., Fuss, I.J., Nieuwenhuis, E.E., Blumberg, R.S. \& Strober, W. Oxazolone colitis, a Th2 colitis model resembling ulcerative colitis, is mediated by IL-13-producing NK-T cells. Immunity 17, 629-638 (2002).

104. Nieuwenhuis, E.E. et al. Disruption of T helper 2 -immune responses in Epstein-Barr virus-induced gene 3-deficient mice. Proc. Natl Acad. Sci. USA 99, 16951-16956 (2002).

105. Saubermann, L.J. et al. Activation of natural killer T cells by alpha-galactosylceramide in the presence of CD1d provides protection against colitis in mice. Gastroenterology 119, 119-128 (2000).

106. Ueno, Y. et al. Single dose of $\mathrm{OCH}$ improves mucosal Thelper type $1 / \mathrm{T}$ helper type 2 cytokine balance and prevents experimental colitis in the presence of valpha14 natural killer T cells in mice. Inflamm. Bowel Dis. 11, 35-41 (2005).

107. Kronenberg, M. \& Gapin, L. The unconventional lifestyle of NKT cells. Nat. Rev. Immunol. 2, 557-568 (2002).

108. Laouar, A. et al. CD70+ antigen-presenting cells control the proliferation and differentiation of T cells in the intestinal mucosa. Nat. Immunol. 6, 698-706 (2005).

109. Niess, J.H. et al. CX3CR1-mediated dendritic cell access to the intestinal lumen and bacterial clearance. Science 307, 254-258 (2005).

110. Jang, M.H. et al. CCR7 is critically important for migration of dendritic cells in intestinal lamina propria to mesenteric lymph nodes. J. Immunol. 176, 803-810 (2006).

111. Johansson-Lindbom, B. et al. Functional specialization of gut CD103+ dendritic cells in the regulation of tissue-selective T cell homing. J. Exp Med. 202, 1063-1073 (2005).

112. Worbs, T. et al. Oral tolerance originates in the intestinal immune system and relies on antigen carriage by dendritic cells. J. Exp. Med. 203, 519-527 (2006).

113. Nagarajan, N.A. \& Kronenberg, M. Invariant NKT cells amplify the innate immune response to lipopolysaccharide. J. Immunol. 178, 2706-2713 (2007).

114. Van Kaer, L. NKT cells: T lymphocytes with innate effector functions. Curr. Opin. Immunol. 19, 354-364 (2007).

115. Germain, R.N. An innately interesting decade of research in immunology. Nat. Med. 10, 1307-1320 (2004). 
116. Brigl, M., Bry, L., Kent, S.C., Gumperz, J.E. \& Brenner, M.B. Mechanism of CD1d-restricted natural killer T cell activation during microbial infection. Nat. Immunol. 4, 1230-1237 (2003).

117. Wu, D. et al. Bacterial glycolipids and analogs as antigens for CD1drestricted NKT cells. Proc. Natl Acad. Sci. USA 102, 1351-1356 (2005).

118. Belperron, A.A., Dailey, C.M. \& Bockenstedt, L.K. Infection-induced marginal zone B cell production of Borrelia hermsii-specific antibody is impaired in the absence of CD1d. J. Immunol. 174, 5681-5686 (2005).

119. Galli, G. et al. CD1d-restricted help to B cells by human invariant natural killer T lymphocytes. J. Exp. Med. 197, 1051-1057 (2003).

120. Mizoguchi, A., Mizoguchi, E., Takedatsu, H., Blumberg, R.S. \& Bhan, A.K. Chronic intestinal inflammatory condition generates IL-10producing regulatory $\mathrm{B}$ cell subset characterized by CD1d upregulation. Immunity 16, 219-230 (2002).

121. Velazquez, P. et al. Villous B cells of the small intestine are specialized for invariant NK T cell dependence. J. Immunol. 180, 4629-4638 (2008).

122. Park, S.H., Benlagha, K., Lee, D., Balish, E. \& Bendelac, A. Unaltered phenotype, tissue distribution and function of Valpha14(+) NKT cells in germ-free mice. Eur. J. Immunol. 30, 620-625 (2000).

123. Hooper, L.V. et al. Molecular analysis of commensal host-microbial relationships in the intestine. Science 291, 881-884 (2001).

124. Artis, D. Epithelial-cell recognition of commensal bacteria and maintenance of immune homeostasis in the gut. Nat. Rev. Immunol. 8, 411-420 (2008).

125. Macpherson, A.J. \& Uhr, T. Induction of protective IgA by intestinal dendritic cells carrying commensal bacteria. Science 303, 1662-1665 (2004).

126. Akira, S., Takeda, K. \& Kaisho, T. Toll-like receptors: critical proteins linking innate and acquired immunity. Nat. Immunol. 2, 675-680 (2001).

127. Ouellette, A.J. Paneth cell alpha-defensin synthesis and function. Curr. Top. Microbiol. Immunol. 306, 1-25 (2006).

128. Ouellette, A.J. Paneth cell alpha-defensins: peptide mediators of innate immunity in the small intestine. Springer Semin. Immunopathol. 27, 133-146 (2005).

129. Salzman, N.H., Underwood, M.A. \& Bevins, C.L. Paneth cells, defensins, and the commensal microbiota: a hypothesis on intimate interplay at the intestinal mucosa. Semin. Immunol. 19, 70-83 (2007).

130. Lacasse, J. \& Martin, L.H. Detection of CD1 mRNA in Paneth cells of the mouse intestine by in situ hybridization. J. Histochem. Cytochem. 40, 1527-1534 (1992).
131. Balk, S.P. et al. Oligoclonal expansion and CD1 recognition by human intestinal intraepithelial lymphocytes. Science $253,1411-1415$ (1991).

132. Perera, L. et al. Expression of nonclassical class I molecules by intestinal epithelial cells. Inflamm. Bowel Dis. 13, 298-307 (2007).

133. Lee, P.T., Benlagha, K., Teyton, L. \& Bendelac, A. Distinct functional lineages of human V(alpha)24 natural killer T cells. J. Exp. Med. 195, 637-641 (2002).

134. Gansert, J.L. et al. Human NKT cells express granulysin and exhibit antimycobacterial activity. J. Immunol. 170, 3154-3161 (2003).

135. Barral, P. et al. B cell receptor-mediated uptake of CD1d-restricted antigen augments antibody responses by recruiting invariant NKT cell help in vivo. Proc. Natl Acad. Sci. USA 105, 8345-8350 (2008).

136. Hampe, J. et al. A genome-wide association scan of nonsynonymous SNPs identifies a susceptibility variant for Crohn disease in ATG16L1. Nat. Genet. 39, 207-211 (2007).

137. Kaser, A. et al. XBP1 links ER stress to intestinal inflammation and confers genetic risk for human inflammatory bowel disease. Cell 134, 743-756 (2008).

138. Marks, D.J. et al. Defective acute inflammation in Crohn's disease: a clinical investigation. Lancet 367, 668-678 (2006).

139. Rioux, J.D. et al. Genome-wide association study identifies new susceptibility loci for Crohn disease and implicates autophagy in disease pathogenesis. Nat. Genet. 39, 596-604 (2007).

140. Wakao, H. et al. A novel mouse model for invariant NKT cell study. J. Immunol. 179, 3888-3895 (2007).

141. Taniguchi, M. et al. Essential requirement of an invariant $\mathrm{V}$ alpha $14 \mathrm{~T}$ cell antigen receptor expression in the development of natural killer $\mathrm{T}$ cells. Proc. Natl Acad. Sci. USA 93, 11025-11028 (1996).

142. Capone, M. et al. Human invariant $V$ alpha $24-J$ alpha $Q T C R$ supports the development of CD1d-dependent NK1.1+ and NK1.1 - T cells in transgenic mice. J. Immunol. 170, 2390-2398 (2003).

143. Cui, J. et al. Requirement for Valpha14 NKT cells in IL-12-mediated rejection of tumors. Science 278, 1623-1626 (1997).

144. Chen, Y.H., Chiu, N.M., Mandal, M., Wang, N. \& Wang, C.R. Impaired NK1 + T cell development and early IL-4 production in CD1-deficient mice. Immunity 6, 459-467 (1997).

145. Smiley, S.T., Kaplan, M.H. \& Grusby, M.J. Immunoglobulin E production in the absence of interleukin-4-secreting CD1-dependent cells. Science 275, 977-979 (1997). 